\title{
On the Output Regulation Problem: The Generalized Second-Order Underactuated Linear System Case
}

\author{
Carlos Aguilar-Ibanez ${ }^{(D)},{ }^{1}$ Jesus A. Meda-Campana, ${ }^{2}$ Miguel S. Suarez-Castanon (D), \\ Jose de Jesus Rubio, ${ }^{4}$ and Nareli Cruz-Cortes ${ }^{1}$ \\ ${ }^{1}$ Instituto Politecnico Nacional, Centro de Investigacion en Computacion, Av. Juan de Dios Batiz s/n, U.P.A.L.M, \\ Ciudad de Mexico 07738, Mexico \\ ${ }^{2}$ Instituto Politecnico Nacional, SEPI-ESIME Zacatenco, Av. Instituto Politecnico Nacional s/n, U.P.A.L.M, \\ Ciudad de Mexico 07738, Mexico \\ ${ }^{3}$ Instituto Politecnico Nacional, Escuela Superior de Computo, Av. Juan de Dios Batiz s/n, U.P.A.L.M., Ciudad de Mexico 07738, Mexico \\ ${ }^{4}$ Instituto Politecnico Nacional, ESIME Azcapotzalco, Av. de las Granjas 682, Ciudad de Mexico 02250, Mexico \\ Correspondence should be addressed to Miguel S. Suarez-Castanon; sasuarez@prodigy.net.mx
}

Received 7 March 2018; Revised 10 September 2018; Accepted 14 October 2018; Published 30 October 2018

Academic Editor: Konstantinos Karamanos

Copyright ( 2018 Carlos Aguilar-Ibanez et al. This is an open access article distributed under the Creative Commons Attribution License, which permits unrestricted use, distribution, and reproduction in any medium, provided the original work is properly cited.

In this work, we aimed to solve the control regulation problem for a generalized second-order underactuated linear system in order to induce a periodic or chaotic behavior or to cancel the external perturbations, generated by an exogenous system, in the nonactuated coordinate. Further, we showed that, in some cases, it is possible to bring to zero the regulation output errors of the underactuated linear plant, depending on the structure of the plant itself and the exogenous system. In the first stage, the solution was developed for the ideal scenario, in which the whole states of the plant and of the exogenous system were available. Secondly, we showed that in some cases it was possible to solve the regulation output problem when only the observable plant output was measurable. That is, the whole plant state and the exogenous signal could be recovered, if some assumptions were fulfilled. The Lyapunov method was used to perform the stability analysis. The proposed solution was assessed through numerical simulations.

\section{Introduction}

In actual control applications, it is common to deal with external perturbations that affect the input of the system or the plant to be controlled. Usually, these perturbations are generated by a different external source, a so-called exogenous system. Due to the importance of this issue, a theory to deal with it has been developed. In this scenario, when the plant has to follow a trajectory, the problem is referred to as the output regulation control problem. Particularly, the exogenous system must exhibit an oscillatory behavior. In more rigorous terms, the regulation problem consists of finding a state or error feedback controller, such that asymptotic stability of the closed-loop system can be achieved, if the external exogenous signal is absent; however, in the presence of the perturbation signal generated by the exogenous system, error tracking convergence to zero can be accomplished (for a detailed treatment of this topic, please refer to [1]).

We outline here the most remarkable works related to this topic. The pioneering work [2] introduced a solution to the linear regulation problem. The same paper introduced the important notion of the linear robust regulator (a controller capable of preserving the regulation property, even when the system parameters are time variant). The authors also showed that, for this case, the linear robust regulation may be achieved, if the controller contains an internal model of the exogenous system. In $[3,4]$, the regulation problem was studied for the class of nonlinear systems with constant exogenous signal. Isidori and Byrnes, in [5], studied the output regulation problem of nonlinear systems with time-varying exogenous signals. Additionally, in this work, the authors probed whether a nonlinear regulator can be obtained on the basis of a set of partial differential equations, 
known as the FIB equations, named after Francis, Isidori, and Byrnes. A similar work was presented by Huang and Rugh in [6], where multivariable nonlinear plants with measured and unmeasured disturbance signals where considered. The following are some of the most important works related to robust output regulation: [7-12]. Finally, several control strategies for the output regulation problem can be found in the literature; however, many of them work with mathematical model approximations or switching the local models using techniques like fuzzy control $[1,13,14]$. A full review of this matter is beyond the scope of this work; however, we suggest to the interested reader the book [15].

The control regulation problem for a generalized secondorder underactuated linear system perturbed by an external signal is studied in this work from the perspective of the regulation theory. The output regulation problem has been solved in the case when the system is fully actuated. Here, we are interesting in the instance where the system is not fully actuated (i.e., where there are fewer actuators than degrees of freedom). Salient to this work we found that notfully actuated systems can be partially regulated, depending on the nature of both the plant and the exogenous system, and the interconnection matrix. Even more, we provide the necessary conditions to accomplish the output regulation for this kind of system. In other words, we were able to assure that the regulation errors $e_{q}=q-\Pi w \rightarrow 0$ and $e_{p}=p-\Pi \dot{w} \longrightarrow 0$, where $q$ and $p$ are the generalized measurable position and the generalized velocity, respectively, $w$ is the exogenous system signal, and $\Pi$ is a suitable constant matrix, susceptible to being designed with some degree of freedom. We underscore that the perturbation signal $w$ has a periodic, quasi-periodic, or chaotic behavior. We must note that, if the regulation errors go to zero, we can induce, evidently, either a chaotic or a periodic behavior, and in some cases we can cancel the effect of $w$ in any of the coordinates, depending on the nature of the matrix $\Pi$. The corresponding stability analysis was accomplished using the Lyapunov theory. It is worth mentioning that the obtained results can be extended to underactuated nonlinear systems, if they are locally controllable and the exogenous signal $w$ is sufficiently small. We numerically verified the effectiveness of the proposed solution using, as testbeds, the Vibrating Mechanical System (VMS) and the Inverted Pendulum Cart System (IPCS), the latter perturbed by small exogenous signals, whose time derivatives were also small. As outcomes we were able to induce a periodic and a chaotic behavior and cancel the perturbation signal in the nonactuated coordinate, even when the perturbation acted solely in the nonactuated acceleration.

At this point, it is important to remark that, in [16], a nonlinear regulator designed on the basis of fuzzy models was applied on the pendubot, which is an underactuated system. However, in that work the regulator was designed supposing that the plant and exosystem's whole state was available (full information case). Besides, perturbations were not considered, and the dimension of the input was equal to the dimension of the output. Therefore, the results obtained in the present work may help to increase the applicability range of the regulation theory on underactuated systems.
The remainder of this work is organized as follows. In Section 2, we establish the output regulation for a generalized second-order underactuated linear system. In Section 3, we solve the established control problem in its ideal case and introduce the main proposition of this work; we also give an extension related to the main proposition, where we deal with the instance in which the state is partially known. The numerical simulations for assessing the effectiveness of the proposed solution are presented in Section 4. Concluding remarks are given in Section 5.

\section{Formulation Control Problem}

Let us consider the following generalized second-order underactuated linear system, modeled as

$$
\left[\begin{array}{c}
\dot{q} \\
\dot{p}
\end{array}\right]=\overbrace{\left[\begin{array}{cc}
0_{n \times n} & I_{n} \\
K_{0} & K_{1}
\end{array}\right]}^{A}\left[\begin{array}{c}
q \\
p
\end{array}\right]+\overbrace{\left[\begin{array}{c}
0_{n \times m} \\
G
\end{array}\right]}^{B} u+\overbrace{\left[\begin{array}{c}
0_{n \times d} \\
P
\end{array}\right]}^{B_{P}} w,
$$

where $q$ and $p \in \mathfrak{R}^{n \times 1}$ are the states; $u \in \mathfrak{R}^{m \times 1}$ is the control input with $m<n ; K_{0}, K_{1} \in \mathfrak{R}^{n \times n}, G \in \mathfrak{R}^{n \times m}$, and $P \in \mathfrak{R}^{n \times d}$ are fixed constant matrices; and $w$ is the external signal generated by a chaotic or quasi periodic exogenous system, defined by

$$
\dot{w}=f(w),
$$

where $f(w) \in \mathfrak{R}^{d \times 1}$ is a smooth function. The control objective consists of designing a controller $u$, formed by

$$
u=K_{q} q+K_{p} p+L(w)
$$

where constants $K_{q}, K_{p} \in \Re^{m \times n}$, and $L(w) \in \Re^{m \times d}$, such that the regulation errors

$$
\begin{aligned}
& e_{q}=q-\Pi w \\
& \dot{e}_{q}=p-\Pi f(w)
\end{aligned}
$$

converge to zero for any initial condition, whereby the constant matrix $\Pi \neq 0$ is conveniently fixed.

The aforementioned problem is closely related to the linear regulator theory (LRT), widely studied in [5, 17-19]. The main differences between these two problems are as follows: (i) the LRT was formulated assuming that the signals $u$ and $e_{q}$ have the same dimensions. In our case, we studied the instance when the dimension of $u$ is strictly less than the dimension of $e_{q}$. That is, $\operatorname{rank}[G]=m<n$; (ii) the LRT was formulated under the assumption that the exogenous system is linear (2). Conversely, in our approach the exosystem $\dot{w}=$ $f(w)$ can be any attractor belonging in a compact set.

Motivation: from our point of view, this control problem has at least two relevant applications in engineering.

First application: for inducing a chaotic or quasi periodic behavior.

It is an easy way to induce oscillations, either quasiperiodic or chaotic, in some class of underactuated flat systems, or for locally controllable nonlinear underactuated 
systems. As we seen from equation (4), if $e_{q}$ and $e_{p}=\dot{e}_{q}$ converge to zero, then, in the steady state, the position and velocity vectors are forced to exhibit a quasi-periodic or chaotic behavior, according to the characteristics of the signal $w$.

Second application: for attenuating disturbances.

It consists of reducing the effect of the external signal $P w$ through the control signal $u$. In other words, we want to attenuate, as much as possible, the effect of $w$ in the system (1) through the action of the controller (3), avoiding the necessity of using either a high-gain controller or a slidingmode controller. In fact, the control action of $u$ forces the convergence to zero of the regulation error:

$$
e=\left[\begin{array}{ll}
e_{q} & e_{p}
\end{array}\right] \longrightarrow 0
$$

keeping the system stable. Notice that if $e \longrightarrow 0$, then, in the steady state, the following inequality is fulfilled:

$$
\begin{gathered}
\|q\| \leq\|\Pi\|\|w\| \\
\|p\| \leq\|\Pi f(w)\| .
\end{gathered}
$$

Hence, selecting the smallest value of all possible values of the norm of $\Pi$, we can assure that the effect of $w$ will be smaller on the states $q$ and $p$. For instance, if $f(w)=S w$, with $\lambda\{S\} \subset \overline{C^{+}}$, then it is easy to see that the following inequalities hold:

$$
\begin{gathered}
\|q\| \leq \xi_{1}\|w\| ; \\
\|p\| \leq \xi_{2}\|w\|,
\end{gathered}
$$

with $\xi_{1}=\|\Pi\|$ and $\xi_{2}=\|\Pi S\|$. That is, the smaller $\xi_{1}$ is, the smaller $q$ and $p$ are. In the developments below we give some conditions to find the matrix $\Pi$ that assures that $e \longrightarrow 0$.

Please note that, in model (1), it is not possible to completely eliminate the effect produced by $w$ due to two facts: first, the matrix $G$ is not invertible, since $\operatorname{rank}[G]=m<$ $n$; second, $\left[0_{n}, P w\right]$ is an unmatched perturbation.

We end this section by introducing some useful assumptions:

(A1) The par $(A, B)$ is stabilizable.

(A2) The constant matrices of system (1) and the structure of $f(w)$ are known.

(A3) For the exogenous system (2), we have that $w, f(w)$, and $\nabla_{w} f(w)$ are well defined and bounded for all future time.

\section{Problem Development}

In this section, we first solve the above-established control problem in its ideal case, in which the states $(q, p, w)$ are always available for measurements. Afterwards, we show that this problem can also be solved using our scheme, when only the state $q$ is measurable and $p$ can always be estimated. Even more, if the interconnection matrix $P$ is not singular, it is possible to recover the states $(p, w)$, provided that only $q$ is available for measurement. Notice that $q$ is the positions vector of the linear underactuated system.
3.1. Solving the Output Regulation Problem for the Underactuated Linear System. First of all, the closed-loop system, defined by (1) and (3), can be rewritten as

$$
\begin{aligned}
& \dot{q}=p \\
& \dot{p}=\left(K_{0}+G K_{q}\right) q+\left(K_{1}+G K_{p}\right) p+G L(w)+P w .
\end{aligned}
$$

Therefore, the time derivative of the regulation error $e$, defined in (4), satisfies the following equation:

$$
\dot{e}=A_{k}\left[\begin{array}{l}
q \\
p
\end{array}\right]+\left[\begin{array}{c}
-\Pi f(w) \\
G L(w)+P w-\Pi \frac{\partial f}{\partial w} f(w)
\end{array}\right],
$$

with

$$
A_{k}=\left[\begin{array}{cc}
0 & I \\
K_{0}+G K_{q} & K_{1}+G K_{p}
\end{array}\right] .
$$

Substituting the values $q=e_{q}+\Pi w$ and $p=e_{p}+\Pi f(w)$, obtained from definition (4), into (11), we obtain:

$$
\dot{e}=A_{k} e+\left[\begin{array}{c}
0 \\
G W_{1}(w)+W_{2}(w)
\end{array}\right],
$$

where

$$
\begin{aligned}
& W_{1}(w)=K_{q} \Pi w+K_{p} \Pi f(w)+L(w) \\
& W_{2}(w)=K_{0} \Pi w+P w+K_{1} \Pi f(w)-\Pi \frac{\partial f}{\partial w} f(w) .
\end{aligned}
$$

To assure that $e$ asymptotically and exponentially converges to zero, we first select $K_{q}$ and $K_{p}$, such that $A_{k}$ becomes a Hurwitz matrix. This fact can always be fulfilled according to (A1). Next, the variables $\Pi$ and $L(w)$ must be selected, such that

$$
G W_{1}(w)+W_{2}(w)=0
$$

Indeed, if (13) is fulfilled and $A_{k}$ is Hurwitz, then the error $e$ goes to zero. Next, to derive the conditions to obtain $L(w)$ and $\Pi$ needed to satisfy the above equation, we multiply both sides of (13) by the following nonsingular matrix:

$$
M=\left[\begin{array}{c}
G^{\perp} \\
G^{T}
\end{array}\right]
$$

where $G^{\perp}$ is the left annihilator of $G$ (that is, $G^{\perp} G=0$ ). The latter leads to the following implications:

$$
0=G^{\perp} W_{2} \text {, }
$$

and

$$
G^{T} G\left(K_{q} \Pi w+K_{p} \Pi f(w)+L(w)\right)+G^{T} W_{2}(w)=0 .
$$

which leads to the following equation:

$$
L(w)=-K_{q} \Pi w-K_{p} \Pi f(w)-G_{+} W_{2}(w),
$$


where $G_{+}=\left(G^{T} G\right)^{-1} G^{T}$. We pointed out that $\Pi$ must be chosen, such that (15) is fulfilled for any $w$. Now, if we found such $\Pi$, then $L(w)$ can be straightforwardly obtained through (17), allowing us to claim that the regulation error, $e$, converges to zero. Finally, according to relation (4), the following holds:

$$
\|q\| \leq\left\|\begin{array}{c}
e_{q} \\
e_{p}
\end{array}\right\|+\left\|\begin{array}{c}
\Pi w \\
\Pi f(w)
\end{array}\right\| .
$$

In other words, in the steady state the following inequalities also holds:

$$
\begin{aligned}
& \|q\| \leq\|\Pi\|\|w\| \\
& \|p\| \leq\|\Pi\|\|f(w)\| .
\end{aligned}
$$

We summarize the previous discussion in the form of the following proposition.

Proposition 1. Consider the interconnected systems (1) and (2), in closed-loop with (3), and under the assumptions (A1), (A2), and (A3). Then, the regulation error e can be accomplished if there exists a matrix $\Pi$ that satisfies the matching equation (15).

Comment 1. Concerning (15), we pointed out that, if $f(w)=$ $S w$, with $S$ being a constant matrix and $\lambda\{S\} \subset \overline{C^{+}}$, then equation (15) leads to

$$
G^{\perp}\left(K_{0} \Pi+P+K_{1} \Pi S-\Pi S^{2}\right)=0 .
$$

It is worth mentioning that, in general, given

$$
\left\{P, K_{0}, K_{1}, G, f(w)\right\} \text {, }
$$

for systems (1) and (2), there does not always exist a matrix $\Pi$ that satisfies the matching equation (15); besides, it is not unique. Then, there are two possibilities, which we analyze below.

(A) $\Pi$ can be selected to solve the disturbances attenuation problem, adding the following condition:

$$
\min _{\Pi \in \Gamma}\|\Pi\|
$$

where $\Gamma$ is the admissible set, defined by

$$
\begin{aligned}
\Gamma= & \left\{\Pi \in \mathfrak{R}^{n \times d}:\right. \\
& G^{\perp}\left(K_{0} \Pi w+P w+K_{1} \Pi f(w)-\Pi \frac{\partial f}{\partial w} f(w)\right)=0, \\
& \forall w \neq 0\} .
\end{aligned}
$$

(B) $\Pi$ is accomplished provided that the nonactuated coordinate of $q$ converges to zero. That is, it is possible to completely eliminate the effects of the exogenous signal $w$ over the nonactuated coordinate of the state $q$.
Comment 2. If there exists a matrix $\Pi$ different from zero, then, according to the inequality (19), it can be possible to induce in the states $q$ and $p$ either a chaotic or a periodic behavior, depending on the nature of the exogenous system (2).

\section{Solving the Control Problem When the State $q$ Is Available}

In the previous discussion, we solved the output regulation problem for the underactuated linear system (1), when all the states were available for measurements. Now, we consider the case when only the state $q$ is available, which entails two cases: (i) the matrix $P$ is singular, $w$ is available for measurements, and the state $p$ is recovered by means of a simple Luenberger observer; (ii) the matrix $P$ is not singular, and the states $p$ and $w$ are not available. To solve the latter case, a Luenberger observer and a high-gain sliding-mode observer that work simultaneously are designed to estimate asymptotically the states $p$ and $w$.

First Case. $P$ is singular, and $q$ and $w$ are available. To solve this case, we use the following controller:

$$
u=K_{q} q+K_{p} \widehat{p}+L(w),
$$

with $\widehat{p}=z_{2}$ evolving according to the following Luenberger observer:

$$
\left[\begin{array}{l}
\dot{z}_{1} \\
\dot{z}_{2}
\end{array}\right]=A_{0} z+\left[\begin{array}{l}
0 \\
G
\end{array}\right] u+\left[\begin{array}{l}
L_{0} \\
L_{1}
\end{array}\right] e_{o 1}+\left[\begin{array}{c}
0 \\
K_{0} q+P w
\end{array}\right],
$$

where $e_{\mathrm{O}_{1}}=q-z_{1}$ is the observation error of the measurable position, $L_{1}>0$ and $L_{2}>0 \in \Re^{n \times n}$, and

$$
A_{0}=\left[\begin{array}{ll}
0_{n} & I_{n} \\
0_{n} & K_{1}
\end{array}\right]
$$

Defining the observation error as

$$
e_{\mathrm{O}}=\left[e_{\mathrm{O}_{1}}=q-z_{1}, e_{\mathrm{O}_{2}}=p-z_{2}\right]^{T} \text {, }
$$

it is easy to see from (1) and (25) that their dynamics fulfill the following equation:

$$
\dot{e}_{0}=\left[\begin{array}{cc}
-L_{0} & I_{n} \\
-L_{1} & K_{1}
\end{array}\right] e_{0}=A_{L} e_{0} .
$$

Observe that $A_{L}$ is Hurwitz, as long as $L_{1}$ and $L_{2}$ fulfill the following inequalities:

$$
\begin{gathered}
L_{1}-K_{1} L_{0}>0 ; \\
L_{0}-K_{1}>0 .
\end{gathered}
$$

Therefore, according to (28a), we can claim that $\|p-\widehat{p}\|$ exponentially and asymptotically converges to zero. Hence, if $\Pi$ and $L(w)$ are selected according to equations (15) and (17), and following the procedure used to obtain (15), it is easy 
to see that the closed-loop system, defined by (1) and (24), is equivalent to the following equation:

$$
\dot{e}=A_{k} e+\left[\begin{array}{c}
0 \\
\left(K_{1}+G K_{p}\right)(p-\widehat{p})
\end{array}\right] .
$$

Now, since the error $\|p-\widehat{p}\|$ exponentially converges to zero and $A_{k}$ is Hurwitz, we concluded that $e$ also converges exponentially to zero. That is, the output tracking error goes to zero when the state $p$ is estimated through the Luenberger observer (25).

Second Case. $P$ is nonsingular and $q$ is measurable. Here we propose the following controller:

$$
u=K_{q} q+K_{p} \widehat{p}+L(\widehat{w})
$$

where $\widehat{p}$ and $\widehat{w}$ will be proposed, such that $\widehat{p} \longrightarrow p$ and $\widehat{w} \longrightarrow w$, for $t \longrightarrow t_{*}$, with $t_{*}>0$ finite. To implement this controller, we introduce the following assumption: (A4) $P$ is invertible. Hence, the Luenberger observer is proposed as

$$
\left[\begin{array}{l}
\dot{z}_{1} \\
\dot{z}_{2}
\end{array}\right]=A_{0}\left[\begin{array}{c}
z_{1} \\
z_{2}
\end{array}\right]+\left[\begin{array}{l}
0 \\
G
\end{array}\right] u+\left[\begin{array}{l}
L_{0} \\
L_{1}
\end{array}\right] e_{\mathrm{O}_{1}}+\left[\begin{array}{c}
0 \\
K_{0} q
\end{array}\right] \text {, }
$$

with $L_{1}$ and $L_{2}$ fulfilling the conditions in (29). Once again, from (1) and (32), we obtain the following dynamic observation error:

$$
\dot{e}_{\mathrm{O}}=A_{L} e_{\mathrm{O}}+B_{p} w
$$

Evidently, if $A_{L}$ is Hurwitz and $w$ is bounded by (A3), then the solution of (33) satisfies the inequality $\left\|e_{o}(t)\right\| \leq \delta(L, P, \bar{w})$, for all $t>t_{0}>0$. In other words, if (29) is fulfilled, we can assure that the observation error is ultimately bounded and stable. Based on the Luenberger observer (32), we give formulas to explicitly recover $w$ and $p$. To this end, we have that (33) is equivalent to the following:

$$
e_{\mathrm{O}_{2}}=\dot{e}_{\mathrm{O}_{1}}+L_{0} e_{\mathrm{O}_{1}}
$$

and

$$
P w=\left(L_{2}-K_{1}\right) e_{\mathrm{O}_{1}}+K_{2} e_{\mathrm{O}_{2}}+\dot{e}_{\mathrm{O}_{2}} .
$$

From the above, it is easy to see that (35) can be rewritten in terms of $e_{o 1}$ as

$$
P w=\left(L_{1}-K_{1} L_{0}\right) e_{\mathrm{O}_{1}}+\left(L_{0}-K_{1}\right) \dot{e}_{\mathrm{O}_{1}}+\ddot{e}_{\mathrm{O}_{1}} .
$$

Notice that, from the last equation, once again $\left\{e_{\mathrm{O}_{1}}, \dot{e}_{\mathrm{O}_{1}}, \ddot{e}_{\mathrm{O}_{1}}\right\}$ are bounded. Now, if assumption (A4) holds, signal $w$ can be algebraically obtained as

$$
w=P^{-1}\left[\left(L_{1}-K_{1} L_{0}\right) e_{O_{1}}+\left(L_{0}-K_{1}\right) \dot{e}_{O_{1}}+\ddot{e}_{O_{1}}\right] .
$$

Similarly, using (34), the corresponding algebraic formula for $p$ is given by

$$
p=z_{2}+\dot{e}_{\mathrm{O}_{1}}+L_{0} e_{\mathrm{O}_{1}}
$$

Since the time derivatives $\dot{e}_{\mathrm{O}_{1}}$ and $\ddot{e}_{\mathrm{O}_{1}}$ are not available for measurement in (37) and (38), we need to propose the following reconstructors to recover them:

$$
\widehat{p}=z_{2}+v_{2}+L_{0} e_{O_{1}},
$$

and

$$
\widehat{w}=P^{-1}\left[\left(L_{1}-K_{1} L_{0}\right) e_{\mathrm{O}_{1}}+\left(L_{0}-K_{1}\right) v_{2}+v_{3}\right],
$$

where the signals $v_{2}$ and $v_{3}$ are proposed, such that

$$
\begin{aligned}
& \lim _{t \rightarrow t_{*}}\left\|\dot{e}_{O_{1}}-v_{2}\right\| \longrightarrow 0 \\
& \lim _{t \rightarrow t_{*}}\left\|\ddot{e}_{O_{1}}-v_{3}\right\| \longrightarrow 0 .
\end{aligned}
$$

In the next section, we use a high-order sliding-mode exact differentiator to obtain the signals $v_{2}$ and $v_{3}(42)$.

Comment 3. If $v_{2}$ and $v_{3}$ fulfill the condition (41), then we also have that $\widehat{p} \longrightarrow p$ and $\widehat{w} \longrightarrow w$, for $t \longrightarrow t_{*}$, with $t_{*}>0$ finite, assuring the exact recovering of the nonavailable signals $p$ and $w$. On the other hand, if assumption (A4) is not fulfilled, we can only exponentially recover the state $p$, through the Luenberger observer (25).

The sliding-mode high-order differentiator: based on Levant's work [20], we propose the following exact differentiator:

$$
\begin{aligned}
& \dot{v}_{1}=v_{2}-\lambda_{1} M^{1 / 3} \alpha_{1}\left(v_{1}-e_{\mathrm{O}_{1}}\right) \operatorname{sign}\left[v_{1}-e_{\mathrm{O}_{1}}\right], \\
& \dot{v}_{2}=v_{3}-\lambda_{2} M^{1 / 3} \alpha_{2}\left(v_{2}-\dot{v}_{1}\right) \operatorname{sign}\left[v_{2}-\dot{v}_{1}\right], \\
& \dot{v}_{3}=-\lambda_{3} M \operatorname{sign}\left[v_{3}-\dot{v}_{2}\right],
\end{aligned}
$$

where

$$
\begin{aligned}
\alpha_{1}(x) & =\left[\left|x_{1}\right|^{2 / 3},\left|x_{2}\right|^{2 / 3}, \ldots,\left|x_{n}\right|^{2 / 3}\right], \\
\alpha_{2}(x) & =\left[\left|x_{1}\right|^{1 / 2},\left|x_{2}\right|^{1 / 2}, \ldots,\left|x_{n}\right|^{1 / 2}\right], \\
\operatorname{sign}[x] & =\left[\operatorname{sign}\left(x_{1}\right), \ldots, \operatorname{sign}\left(x_{n}\right)\right] .
\end{aligned}
$$

Under the assumption that there exists a constant $C>0$, with $\left\|e_{\mathrm{O}_{1}}^{(i)}(t)\right\| \leq C<M$, for $i=\{0, \ldots, 3\}$, we have that, for some $t_{*}>0$, the following equalities hold:

$$
\begin{aligned}
& \lim _{t \rightarrow t_{*}}\left\|e_{o 1}-v_{1}\right\| \longrightarrow 0 ; \\
& \lim _{t \rightarrow t_{*}}\left\|\ddot{e}_{o 1}-v_{2}\right\| \longrightarrow 0 ; \\
& \lim _{t \rightarrow t_{*}}\left\|\ddot{e}_{o 1}-v_{3}\right\| \longrightarrow 0,
\end{aligned}
$$

as long as the gains $\lambda_{i}$ are selected as

$$
\begin{aligned}
& \lambda_{1}=1.1 M, \\
& \lambda_{2}=1.5 \sqrt{M}, \\
& \lambda_{3}=1.5 M .
\end{aligned}
$$


Comment 4. According to the inequalities in (29), (36), and the fact that $w$ and $f(w)$ are both bounded, we have that $\left\|e_{\mathrm{O}_{1}}^{(i)}(t)\right\| \leq C<M$, for $i=\{0, \ldots, 3\}$, always holds. In fact, the actual value of $C$ cannot be accurately computed, but we can always select some $M>0$ sufficiently large to overcome this inconvenience.

We end this section summarizing the above discussion in the form of the following lemma.

Proposition 2. Consider system (36), with measurable output $q$, under assumption (A3), with $L_{0}$ and $L_{1}$ fulfilling (29). Then, for sufficiently large $M$, and $\lambda_{i}$ selected according to (45), we have that the sliding-mode high-order differentiator (42) assures the conditions in (44). Consequently, the estimation $\widehat{p}$ given in (39) converges to $p$ in finite time. Complementarily, if assumption (A4) is fulfilled, then the estimation of $\widehat{w}$ also converges to $w$ in finite time.

Comment 5. The closed-loop system (9) can be seen as a second-order linear system, perturbed by a bounded signal $w$, with $A_{k}$ exponentially stable. Evidently, as long as the estimations of $\widehat{p}$ and $\widehat{w}$ converge to their corresponding actual values in finite time, all the solutions of the closed-loop system, in combination with the Luenberger observer (32) and the exact differentiator (42), are bounded and well defined, for all future, fulfilling the separation principle. Consequently, the output regulation problem is accomplished.

\section{Numerical Examples}

To illustrate the performance of the proposed solution to the output regulation problem, we designed three numerical experiments. The first two use the underactuated Vibrating Mechanical System (VMS), and the third one uses the traditional Cart-Pendulum system (CPS). For consistency, we consider that both systems are affected by two external vibration signals, $g_{1_{w}}(t)$ and $g_{2_{w}}(t)$, generated through

$$
\left[\begin{array}{l}
g_{1 w}(t) \\
g_{2 w}(t)
\end{array}\right]=P w
$$

where $w=\left[w_{1}, w_{2}\right]^{T}$ is the vector of the exogenous signals.

5.1. Description of VMS for the First Two Experiments. The VMS, depicted in Figure 1, is made up of two coupled subsystems. The secondary subsystem has an active undamped dynamic vibration absorber and is coupled to the primary perturbed system. The dynamics of this system are described by the following set of differential equations:

$$
\begin{aligned}
{\left[\begin{array}{c}
\dot{q}_{1} \\
\dot{q}_{2} \\
\dot{p}_{1} \\
\dot{p}_{2}
\end{array}\right]=} & {\left[\begin{array}{cccc}
0 & 0 & 1 & 0 \\
0 & 0 & 0 & 1 \\
-\frac{k_{1}+k_{2}}{m_{1}} & \frac{k_{2}}{m_{1}} & -\frac{c_{1}}{m_{1}} & 0 \\
\frac{k_{2}}{m_{1}} & -\frac{k_{2}}{m_{2}} & 0 & 0
\end{array}\right]\left[\begin{array}{l}
q_{1} \\
q_{2} \\
p_{1} \\
p_{2}
\end{array}\right]+\left[\begin{array}{l}
0 \\
0 \\
0 \\
1
\end{array}\right] u } \\
& +\left[\begin{array}{c}
0_{2 \times 2} \\
\frac{P}{m_{1}}
\end{array}\right] w
\end{aligned}
$$

where the variables $q_{1}$ and $p_{1}$ are, respectively, the position and the velocity of mass $m_{1}$, in the same way that $q_{2}$ and $p_{2}$ are, respectively, the position and velocity the of mass $m_{2}$. The springs' linear stiffness values are denoted by $k_{1}$ and $k_{2}$, and $c_{1}$ is the viscous damping; $P$ is a constant interconnection matrix; $w \in \Re^{2 \times 1}$ is the vector of the exogenous perturbation signals, generated by some target system and proposed in each experiment; and $u$ represents the system input or control action. Summarizing, $q=\left[q_{1}, q_{2}\right]^{T}, p=\left[p_{1}, p_{1}\right]^{T}$, and $w=$ $\left[w_{1}, w_{2}\right]^{T}$ are, respectively, the positions vector, the velocities vector, and the exogenous signals vector. To simplify the following developments, we fixed the system parameters as $m_{1}=m_{2}=k_{1}=k_{2}=1$. Hence, according to (1), we have that the corresponding characteristic matrices for the VMS are given as

$$
\begin{aligned}
K_{0} & =\left[\begin{array}{cc}
-2 & 1 \\
1 & -1
\end{array}\right] ; \\
K_{1} & =\left[\begin{array}{cc}
-c_{1} & 0 \\
0 & 0
\end{array}\right] ; \\
G & =\left[\begin{array}{l}
0 \\
1
\end{array}\right] .
\end{aligned}
$$

Notice that $q_{2}$ is the actuated coordinate and $q_{1}$ is the nonactuated coordinate.

First Experiment. For this experiment, we assume that an external vibration signal, $g_{1 w}(t)$, is present in the acceleration of the nonactuated coordinate, $q_{1}$ (evidently, $g_{2 w}(t)=0$ for all $t$ ). The goal of this experiment consists of canceling the vibration in the nonactuated coordinate, assuming that the vector position, $q=\left[q_{1}, q_{2}\right]^{T}$, and $w$ are available. In other words, we consider that $g_{1 w}(t)=p_{11} w_{1}+p_{12} w_{2}$ and $g_{2 w}(t)=$ 0 , where $w$ is generated by the following system:

$$
\dot{w}=\left[\begin{array}{cc}
0 & 1 \\
-1 & 0
\end{array}\right] w=S w
$$

Evidently, for this case, the interconnection matrix $P$ is given by

$$
P=\left[\begin{array}{cc}
p_{11} & p_{12} \\
0 & 0
\end{array}\right]
$$

Therefore, the matrix $\Pi \in \mathfrak{R}^{2 \times 2}$ must satisfy, according to (20), the following equation:

$$
\left[\begin{array}{ll}
1 & 0
\end{array}\right]\left(K_{0} \Pi+P+K_{1} \Pi S-\Pi S^{2}\right)=0 .
$$

After substituting the corresponding values of $\left\{K_{0}, K_{1}, S, P\right\}$, given in (48), (49), and (50), into equation (51), we obtain

$$
\Pi=\left[\begin{array}{cc}
\pi_{1} & \pi_{2} \\
\pi_{1}-c_{1} \pi_{2}-p_{11} & c_{1} \pi_{1}+\pi_{2}-p_{12}
\end{array}\right] .
$$

where the parameters $\pi_{1}$ and $\pi_{2}$ must be fixed, such that they assure that $q_{1} \longrightarrow 0$ and $p_{1} \longrightarrow 0$. That is, we 


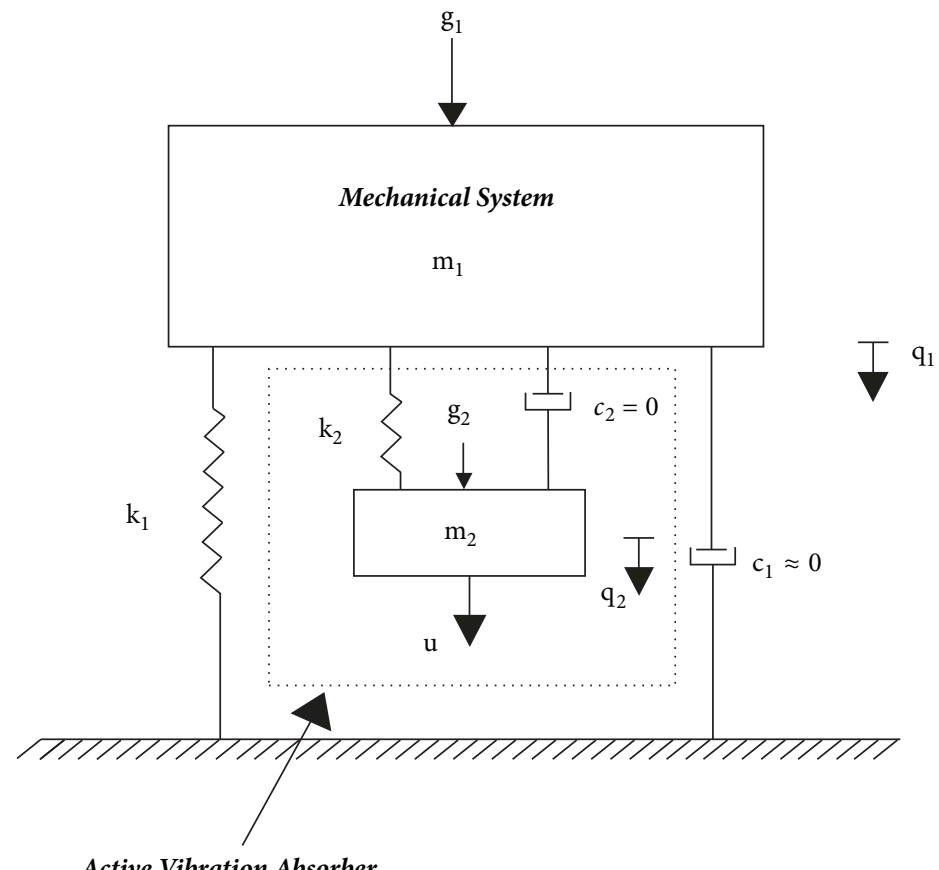

FIgURE 1: The underactuated VMS.
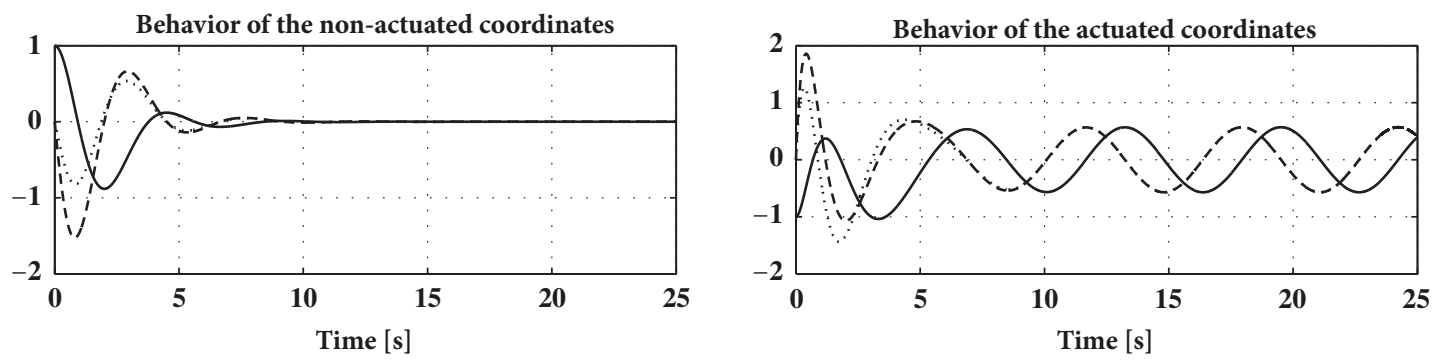

$\begin{array}{ll}- & q_{1} \\ -\cdots \cdot & p_{1} \\ \cdots & \widehat{p}_{1}\end{array}$
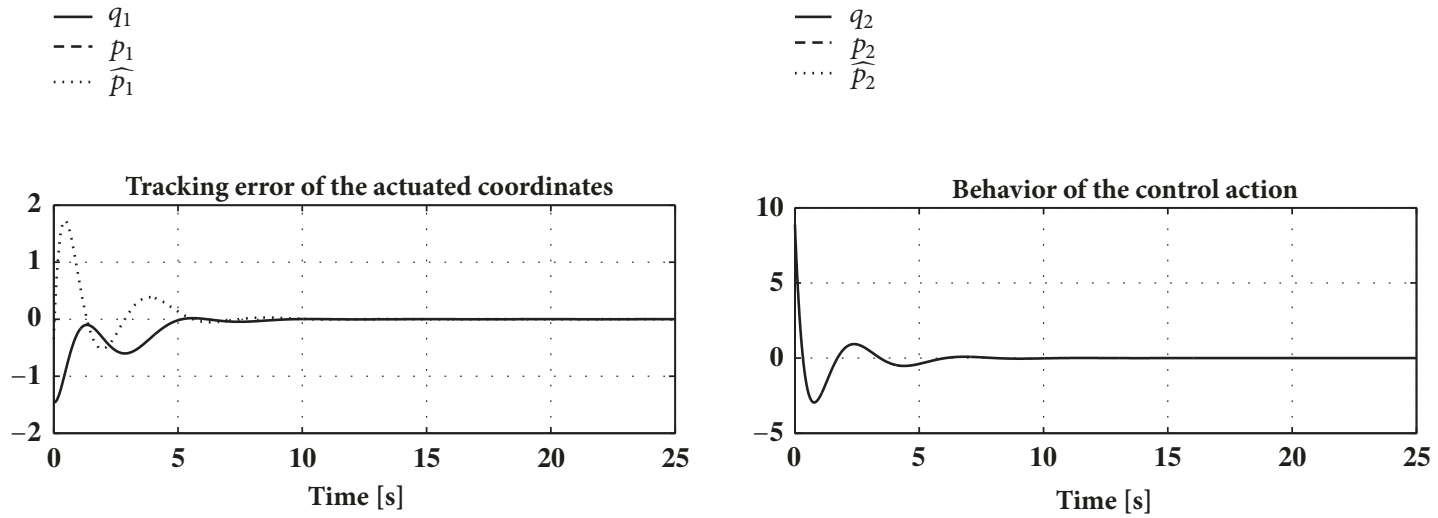

- $e_{q 2}$

$-u$

FIGURE 2: Cancelation of the exogenous signal $g_{1}$ in the perturbed nonactuated coordinate of the VMS.

want to cancel the effect of the exogenous signal $g_{1_{w}}$ in the nonactuated coordinates $q_{1}$ and $p_{1}$. This can be achieved by selecting $\pi_{1}=\pi_{2}=0$ (see equation (4)). To obtain the nonavailable variable $p$, we use the observer (25), where $L_{0}=I_{2}$ and $L_{1}=2 I_{2}$ assure the condition (29). The initial condition for the system is given by $q(0)=[1,-1]$ and $p=0$; the exogenous system was initialized in $w(0)=$ $[0.2,-0.3]$; and the corresponding control gains were set as $K_{q}=[4.156,-2.31]$ and $K_{p}=[4.13,-3.9]^{T}$. In Figure 2, we show the outcome of this experiment, for $c_{1}=0.5, p_{11}=$ 
0.5 , and $p_{12}=1$. In this figure, we can see the closed-loop behavior, the evolution of both the actuated and nonactuated coordinates, and the corresponding errors, $e_{q_{1}}$ and $e_{p_{2}}$. We can also see that $\widehat{p}$ converges to $p$ after 7 seconds, while the errors $e_{q}$ and $e_{p}$ converge to zero after 10 seconds. In addition, we can see that the nonactuated coordinates remain oscillating in steady state after 10 seconds. We can see that the control reaches a steady state at 7 seconds.

Second Experiment. This experiment consists of inducing a chaotic behavior in both the actuated and the nonactuated coordinates of the VMS, through the exogenous signals $g_{1 w} \neq$ 0 and $g_{2 w} \neq 0$, where $w$ is generated through the GenesioTesi attractor (GTA). This control task will be carried out assuming that the state $q$ is available. The GTA $[21,22]$ is described by the following set of equations:

$$
\begin{aligned}
& \dot{w}_{1}=w_{2} ; \\
& \dot{w}_{2}=w_{3} ; \\
& \dot{w}_{3}=-a w_{1}-b w_{2}-c w_{3}+w_{1}^{2},
\end{aligned}
$$

where $a, b$, and $c$ are strictly positive constants, such that $a b<$ $c$, and the interconnection matrix is fixed as

$$
P=\left[\begin{array}{lll}
p_{11} & p_{12} & p_{13} \\
p_{21} & p_{22} & p_{23}
\end{array}\right]
$$

Evidently, matrix $P$ is not invertible and, therefore, does not fulfill assumption (A4). Consequently, the state $w$ cannot be recovered; hence, it has to be measured. It is easy to see that the GTA has the following Jacobian matrix:

$$
\frac{\partial f}{\partial w}=\left[\begin{array}{ccc}
0 & 1 & 0 \\
0 & 0 & 1 \\
-c+2 w_{1} & -b & -a
\end{array}\right] .
$$

For simplicity, we fixed the parameters

$$
\begin{aligned}
& a=1 \\
& b=2 \\
& c=3 \\
& c_{1}=0 .
\end{aligned}
$$

To find the needed matrix $\Pi$, defined as

$$
\Pi=\left[\begin{array}{lll}
\pi_{1} & \pi_{2} & \pi_{3} \\
\pi_{4} & \pi_{5} & \pi_{6}
\end{array}\right]
$$

we substitute the expressions (54), (48), and (57) into the single equation (15), leading to the following nonlinear function:

$$
\alpha_{1} w_{1}^{2}+\alpha_{2} w_{2}+a_{3} w_{1}-2 \pi_{3} w_{1} w_{2}+\alpha_{4} w_{3}=0,
$$

where

$$
\begin{aligned}
& \alpha_{1}=-\pi_{2}+\pi_{3}, \\
& \alpha_{2}=\pi_{3}+\pi_{5}+p_{12} \\
& \alpha_{3}=-2 \pi_{1}+3 \pi_{2}-3 \pi_{3}+\pi_{4}+p_{11}, \\
& \alpha_{4}=-\pi_{1}+\pi_{2}-\pi_{3}+\pi_{6}+p_{13} .
\end{aligned}
$$

To make (58) hold for any $w$, we must assure that $\alpha_{i}=0$, $i=\{1,2,3,4\}$. The latter implies the following:

$$
\Pi=\left[\begin{array}{ccc}
\pi_{6}+p_{13} & 0 & 0 \\
2\left(\pi_{6}+p_{13}\right)-p_{11} & -p_{12} & \pi_{6}
\end{array}\right],
$$

where $\pi_{6}$ is a free parameter. To carry out the second experiment, the gains $L_{0}, L_{1}, K_{q}$ and $K_{p}$ were set as in the first experiment. The parameters of the interconnection matrix $P$ were fixed as $p_{1 i}=0.1$ and $p_{2 i}=-0.2$, for $i=\{1,2,3\}$. The initial condition for the GTA was initialized as $w(0)=$ $[0.6,-0.1,0.1]$, while the VMS was initialized as in the first experiment. The outcome of this experiment is shown in Figure 3, which shows the tracking errors of the actuated and nonactuated coordinates. In the same figure, we show the phase portraits of both coordinates. From this figure, we can see that the error goes very close to zero after 11 seconds for both the actuated and the nonactuated coordinates. Additionally, we can see, from the phase portraits, that the VMS system effectively exhibits a chaotic behavior, even when the simulation time took 150 seconds. It is worth mentioning that the experiments were carried out using a computer simulation environment that uses a sixth-order Runge-Kutta algorithm, with an integration step of $10^{-3}$. This configuration helped us see the chaotic behavior of the GTA. In fact, if we use a greater integration step, we cannot appreciate the chaotic behavior of this system.

5.2. Description of PCS for the Third Experiment. To carry out this experiment, we consider the normalized partially feedback model of the PCS ([23]), described by the following set of differential equations:

$$
\left[\begin{array}{c}
\dot{q}_{1} \\
\dot{q}_{2} \\
\dot{p}_{1} \\
\dot{p}_{2}
\end{array}\right]=\left[\begin{array}{c}
p_{1} \\
p_{2} \\
\sin q_{1}-u \cos q_{1} \\
u
\end{array}\right]+\left[\begin{array}{c}
0_{2 \times 2} \\
P w
\end{array}\right],
$$

where $q_{1}$ is the actual angular position of the pendulum with respect to the vertical, and $q_{2}$ is the normalized horizontal displacement of the cart, while their corresponding angular and horizontal velocities are $p_{1}$ and $p_{2}$, respectively. The virtual controller is defined as $u$, and $P w$ has the same definition as in (46), provided that $w$ is sufficiently small to make the system to oscillate around to the unstable equilibrium point $(q=0, p=0)$.

Third Experiment. This experiment consists of inducing a limit cycle using solely measurements of the pendulum and 

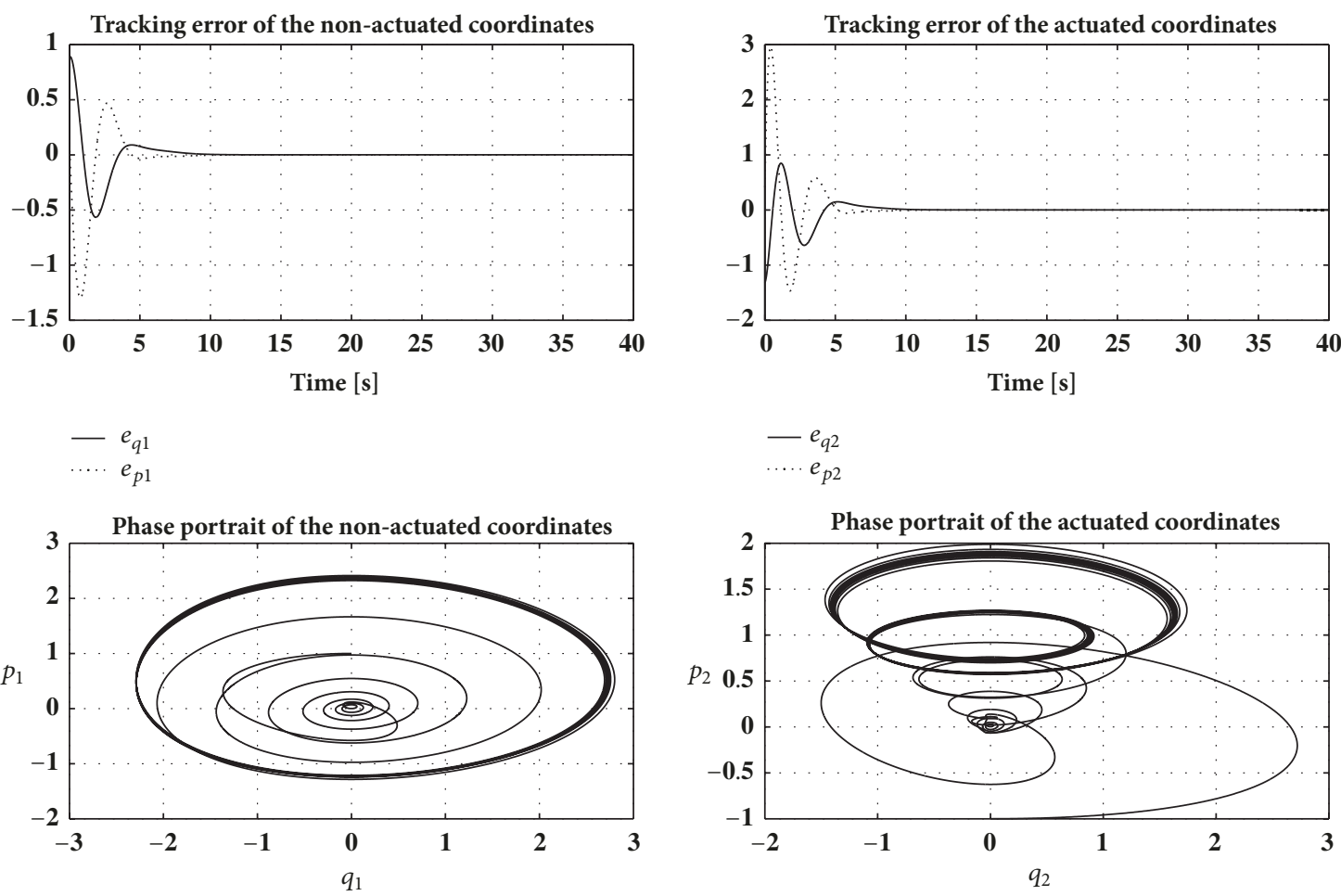

FIGURE 3: Inducing a chaotic behavior in the actuated and nonactuated coordinates of the VMS, through the Genesio-Tesi system.

the cart positions. The limit cycle is produced by the linear exogenous system, defined as

$$
\dot{w}=\left[\begin{array}{cc}
0 & n_{i} \\
-1 & 0
\end{array}\right]=S w,
$$

where $n_{i}$ can be any positive constant, and the interconnection matrix is fixed as $P=\operatorname{diag}\{1,1\}$. In this case, we assume that $q$ is available for measurements and that $p$ will be recovered using the Luenberger observer defined in (32), while $w$ will be recovered using the high-order exact differentiator (42) and the reconstructor (40). To make our methodology adequate, we use the linearized model of the system (61), given by

$$
\left[\begin{array}{l}
\dot{q}_{1} \\
\dot{q}_{2} \\
\dot{p}_{1} \\
\dot{p}_{2}
\end{array}\right]=\left[\begin{array}{llll}
0 & 0 & 1 & 0 \\
0 & 0 & 0 & 1 \\
1 & 0 & 0 & 0 \\
0 & 0 & 0 & 0
\end{array}\right]\left[\begin{array}{c}
q_{1} \\
q_{2} \\
p_{1} \\
p_{2}
\end{array}\right]+\left[\begin{array}{c}
0 \\
0 \\
-1 \\
1
\end{array}\right] u+\left[\begin{array}{c}
0_{2 \times 2} \\
P w
\end{array}\right] .
$$

Mimicking the steps as in the first experiment and, according to the matching equation (20), we have that

$$
\left[\begin{array}{ll}
1 & 1
\end{array}\right]\left(K_{0} \Pi+P-\Pi S^{2}\right)=0
$$

where

$$
\begin{aligned}
K_{0} & =\left[\begin{array}{ll}
1 & 0 \\
0 & 0
\end{array}\right] ; \\
\Pi & =\left[\begin{array}{ll}
\pi_{11} & \pi_{12} \\
\pi_{21} & \pi_{22}
\end{array}\right],
\end{aligned}
$$

with $S$ defined in $(62)$ and $P=\operatorname{diag}\{1,1\}$. Substituting $K_{0}, \Pi$, $P$, and $S$ into equation (64), we obtain the following:

$$
\Pi=\left[\begin{array}{cc}
\frac{-1-n_{i} \pi_{21}}{1+n_{i}} & \pi_{12} \\
\pi_{21} & \frac{-1-\pi_{12}-n_{i} \pi_{12}}{n_{i}}
\end{array}\right],
$$

where $\pi_{12}$ and $\pi_{21}$ can be fixed as desired. To accomplish the goal of this experiment, which is to assure that $q \longrightarrow \Pi w$ and $q \longrightarrow \Pi S w$, we use the following set up. The gains $L_{0}$, $L_{1}, K_{q}$ and $K_{p}$ were fixed as in the first experiment, while $\pi_{12}=0.4, \pi_{21}=0.6$, and $n_{i}=0.5$. The initial condition of the exogenous system was set as $w(0)=[0.1,-0.2]$, and the PCS was initialized at $q=(0.15,-03)$ and $p=0$. Because $P$ is nonsingular, the PCS fulfills the conditions of the assumption (A4). Therefore, we propose the parameter $M=2$, for the sliding-mode high-order differentiator (42). The obtained results can be seen in Figure 4, where we present the tracking errors of the actuated and the nonactuated coordinates. In the same figure, we show the phase portraits of both coordinates. From this figure, we can see that the tracking error goes very close to zero after 11 seconds, for both the actuated and the nonactuated coordinates. Additionally, we can see, from the phase portraits, that the PCS system effectively exhibits a limit cycle in both the pendulum angular coordinate and the cart horizontal coordinate, even when the controller uses the estimated velocity vector $\widehat{p}$ and the reconstructed exogenous perturbation vector $\widehat{w}$. 

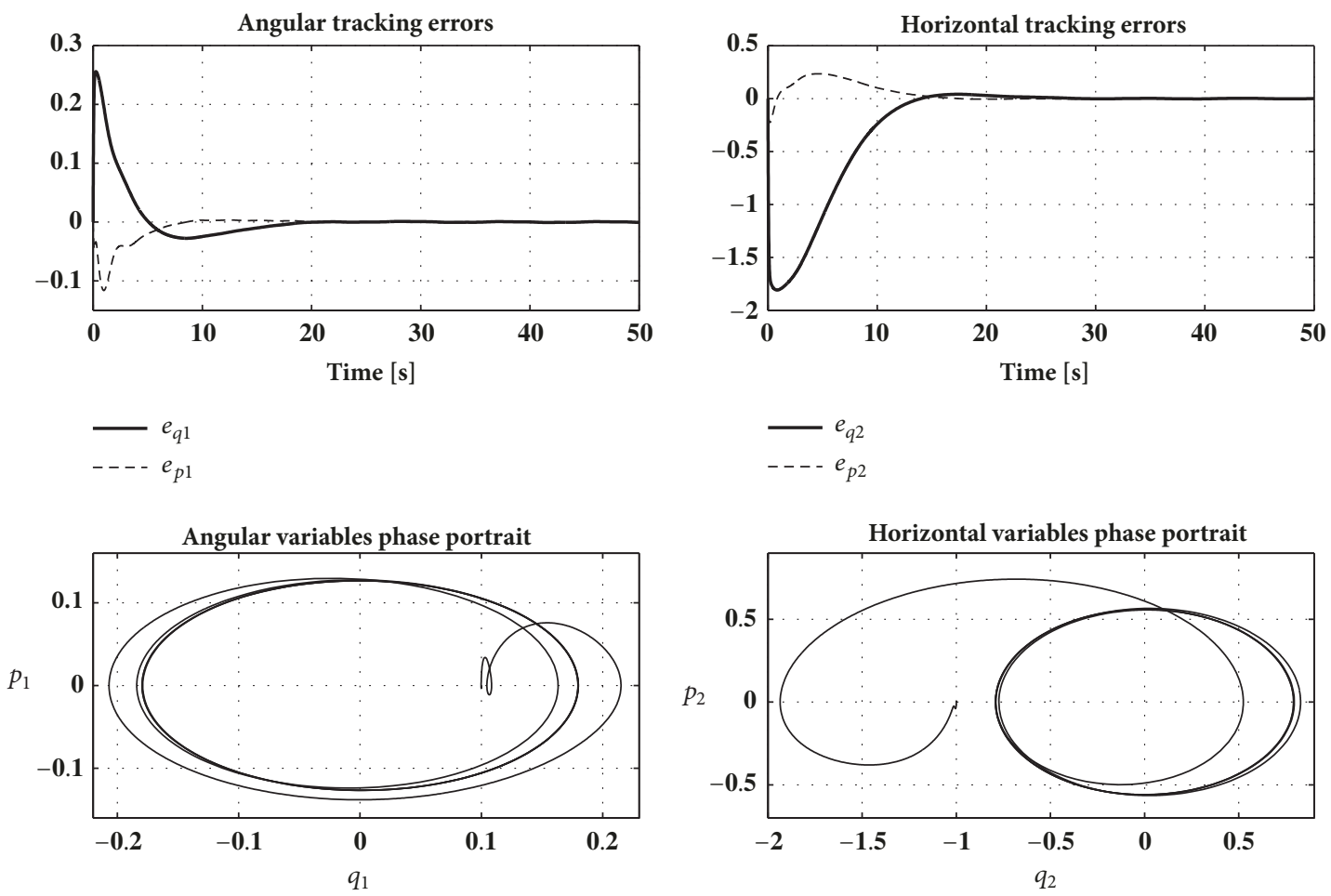

FIGURE 4: Tracking errors for the angular and horizontal positions for the PCS and corresponding phase portraits of these positions.

\section{Concluding Remarks}

In this work, we studied the control regulation problem for a generalized second-order underactuated linear system perturbed by an external signal, generated by a chaotic or quasi-periodic exogenous system. We underscore that the well-known problem can be solved provided that the number of controllers coincides with the number of degrees of freedom. In this study, we analyzed the case in which we have fewer actuators than degrees of freedom. Under this strong assumption, we conclude that, in some cases, it is possible to assure that the regulation errors $\left(e_{q}, e_{p}\right)$, introduced in (4), can be brought to zero relying on the system structure, which in turn depends on the nature of the exogenous system and the interconnection matrix $P$, as we established in Proposition 1. To obtain the solution to the aforementioned problem, we worked with the ideal case, in which all the state variables and the exogenous signal were available for measurements. Then, we relaxed this condition assuming that solely the position vector, $q$, and the exogenous signal, $w$, were available, while the velocity vector, $p$, can be recovered by a Luenberger observer. Besides, if the interconnection matrix $P$ is not singular, we can recover both $p$ and $w$, only based on measurements of $q$. It is worth mentioning that this control scheme, which is based on the linear regulation theory, can be applied to induce either periodic or chaotic behaviors in the underactuated linear system. Also, the scheme allows us to cancel in some cases the effect of the exogenous signal in the nonactuated coordinate, through appropriate control actions. This was illustrated in the first numerical simulation, where we used the VMS, which was subject to a sinusoidal exogenous signal in the nonactuated coordinate, and where the control task consisted of canceling this effect. The corresponding stability analysis was carried out applying the Lyapunov method. Finally, we performed three convincing numerical simulations, where we showed, first, that our control scheme effectively canceled the undesirable exogenous perturbations in the nonactuated coordinate and, second, that a periodic or chaotic behavior was induced.

\section{Data Availability}

No data were used to support this study.

\section{Conflicts of Interest}

The authors declare that they have no conflicts of interest.

\section{Acknowledgments}

This research was supported by the Secretaria de Investigacion y Posgrado del IPN, under Research Grants 20181776 and 20181101.

\section{References}

[1] J. A. Meda-Campaña, J. C. Gómez-Mancilla, and B. CastilloToledo, "Exact output regulation for nonlinear systems described by Takagi-Sugeno fuzzy models," IEEE Transactions on Fuzzy Systems, vol. 20, no. 2, pp. 235-247, 2012.

[2] B. A. Francis and W. M. Wonham, "The internal model principle of control theory," Automatica, vol. 12, no. 5, pp. 457-465, 1976. 
[3] E. J. Davison, "The Robust Control of a Servomechanism Problem for Linear Time-Invariant Multivariable Systems," IEEE Transactions on Automatic Control, vol. 21, no. 1, pp. 2534, 1976.

[4] B. A. Francis and W. M. Wonham, "The internal model principle for linear multivariable regulators," Applied Mathematics and Optimization. An International Journal with Applications to Stochastics, vol. 2, no. 2, pp. 170-194, 1975.

[5] A. Isidori and C. I. Byrnes, "Output regulation of nonlinear systems," IEEE Transactions on Automatic Control, vol. 35, no. 2, pp. 131-140, 1990.

[6] J. Huang and W. J. Rugh, "On a nonlinear multivariable servomechanism problem," Automatica, vol. 26, no. 6, pp. 963972, 1990.

[7] C. I. Byrnes, F. Delli Priscoli, A. Isidori, and W. Kang, "Structurally stable output regulation of nonlinear systems," Automatica, vol. 33, no. 3, pp. 369-385, 1997.

[8] F. Delli Priscoli, "Robust tracking for polynomial plants," in Proceedings of the European Control Conference, pp. 369-373, 1993.

[9] J. Huang, "Asymptotic tracking and disturbance rejection in uncertain nonlinear systems," Institute of Electrical and Electronics Engineers Transactions on Automatic Control, vol. 40, no. 6, pp. 1118-1122, 1995.

[10] J. Huang and C.-F. Lin, "On a robust nonlinear servomechanism problem," Institute of Electrical and Electronics Engineers Transactions on Automatic Control, vol. 39, no. 7, pp. 1510-1513, 1994.

[11] J. Huang and C.-F. Lin, "Internal model principle and robust control of nonlinear systems," in Proceedings of the 32nd IEEE Conference on Decision and Control. Part 2 (of 4), pp. 1501-1506, December 1993.

[12] H.-K. Khalil, "Robust servomechanism output feedback controllers for feedback linearizable systems," Automatica, vol. 30, no. 10, pp. 1587-1599, 1994.

[13] B. Castillo-Toledo and J. A. Meda-Campaña, "The fuzzy discrete-time robust regulation problem: a LMI approach," in Proceedings of the 41st IEEE Conference on Decision and Control, vol. 2, pp. 2159-2164, IEEE, Las Vegas, Nev, USA, December 2002.

[14] N. R. Cazarez-Castro, L. T. Aguilar, and O. Castillo, "Fuzzy logic control with genetic membership function parameters optimization for the output regulation of a servomechanism with nonlinear backlash," Expert Systems with Applications, vol. 37, no. 6, pp. 4368-4378, 2010.

[15] J. C. Doyle, B. A. Francis, and A. R. Tannenbaum, Feedback Control Theory, Courier Corporation, 2013.

[16] J. A. Meda-Campaña, B. Castillo-Toledo, and V. Zúñiga, "On the nonlinear fuzzy regulation for under-actuated systems," in Proceedings of the 2006 IEEE International Conference on Fuzzy Systems, pp. 2195-2202, Canada, July 2006.

[17] B. A. Francis, "The linear multivariable regulator problem," SIAM Journal on Control and Optimization, vol. 15, no. 3, pp. 486-505, 1977.

[18] L. E. Ramos, B. Castillo-Toledo, and J. Alvarez, "Nonlinear regulation of an underactuated system," in Proceedings of the 1997 IEEE International Conference on Robotics and Automation, ICRA. Part 4 (of 4), pp. 3288-3293, April 1997.

[19] B. Castillo-Toledo, S. Celikovsky, and S. Di Gennaro, "Generalized immersion and nonlinear robust output regulation problem," Kybernetika, vol. 40, no. 2, pp. 207-220, 2004.
[20] A. Levant, "Higher-order sliding modes, differentiation and output-feedback control," International Journal of Control, vol. 76, no. 9-10, pp. 924-941, 2003.

[21] A. Luviano-Juarez, J. Cortes-Romero, and H. Sira-Ramirez, "Synchronization of chaotic oscillators by means of generalized proportional integral observers," International Journal of Bifurcation and Chaos, vol. 20, no. 5, pp. 1509-1517, 2010.

[22] A. G. Soriano-Sanchez, C. Posadas-Castillo, M. A. Platas-Garza et al., "Coupling strength computation for chaotic synchronization of complex networks with multi-scroll attractors," Applied Mathematics and Computation, vol. 275, pp. 305-316, 2016.

[23] C. Aguilar-Ibañez, J. C. Martínez-García, A. Soria-López et al., "On the Stabilization of the Inverted-Cart Pendulum Using the Saturation Function Approach," Mathematical Problems in Engineering, vol. 2011, Article ID 856015, 14 pages, 2011. 


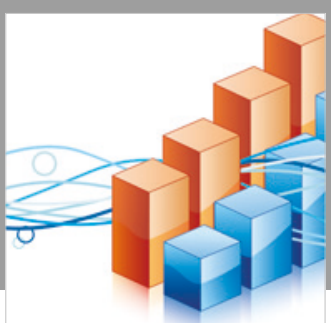

Advances in

Operations Research

\section{-n-m}
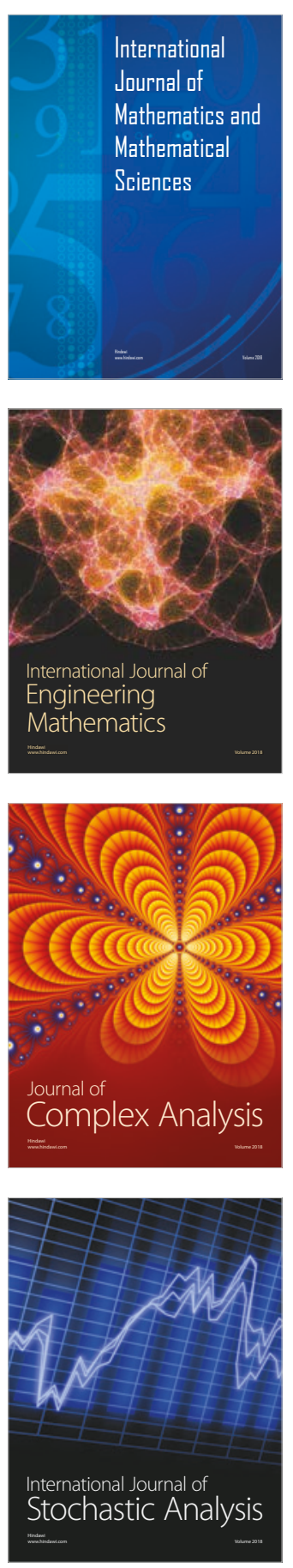
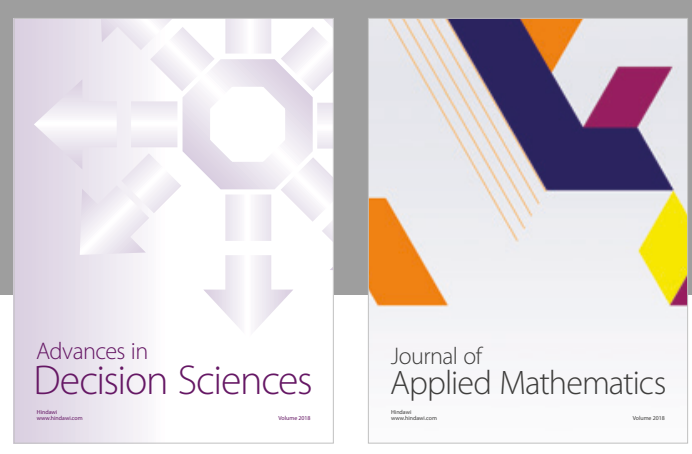

Journal of

Applied Mathematics
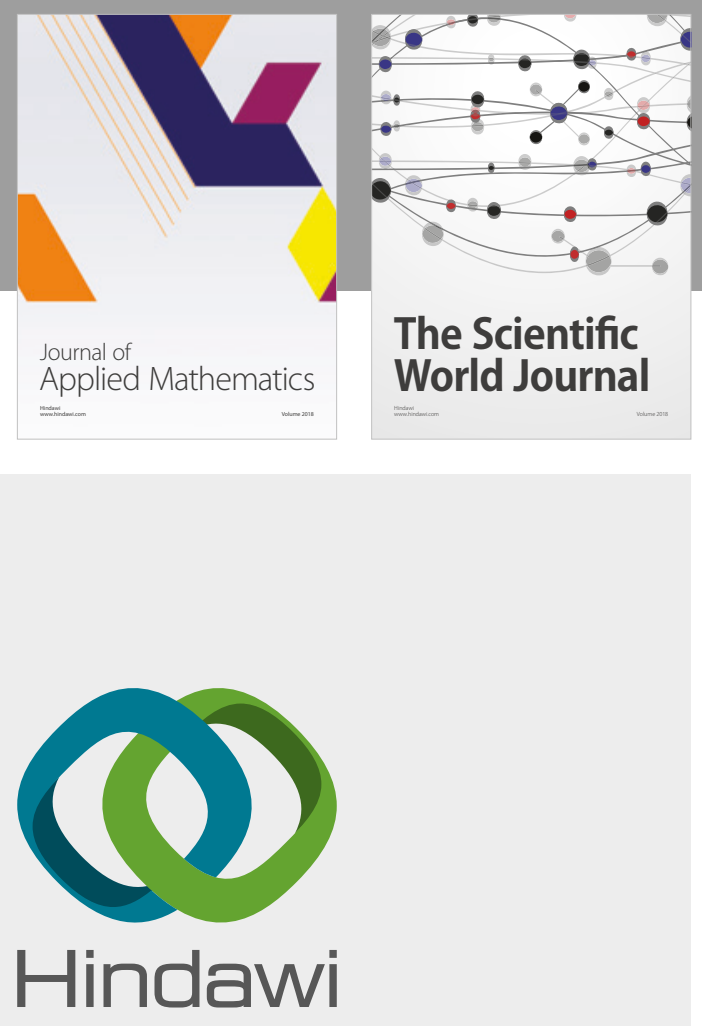

Submit your manuscripts at

www.hindawi.com

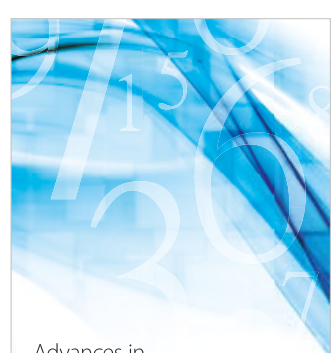

Advances in
Numerical Analysis
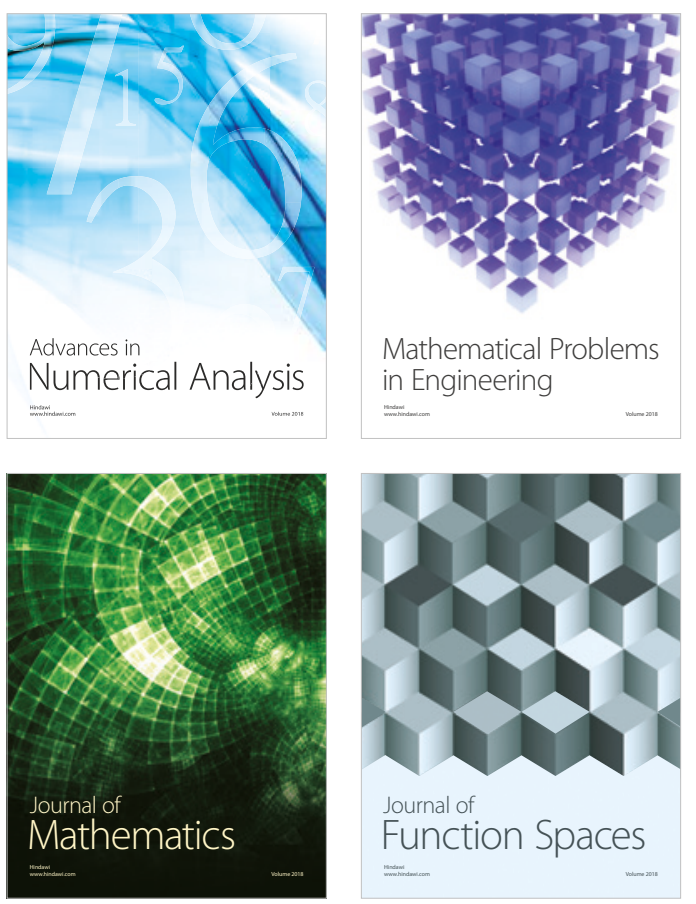

Mathematical Problems in Engineering

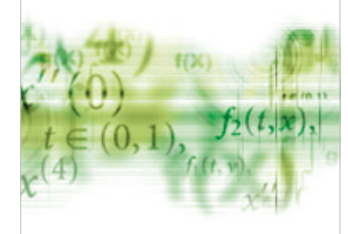

International Journal of

Differential Equations

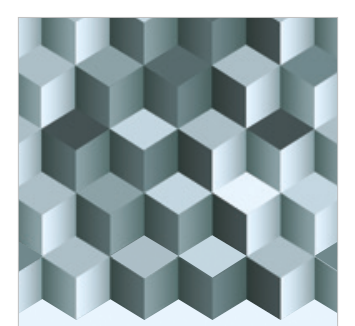

Journal of

Function Spaces

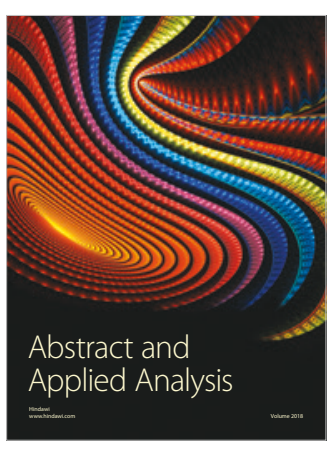

The Scientific

World Journal

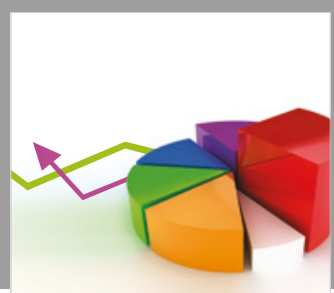

Journal of

Probability and Statistics
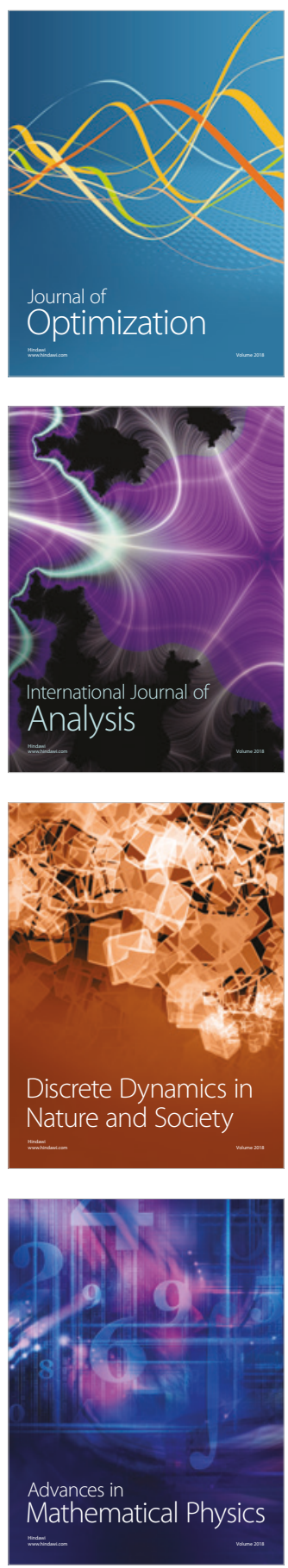